\title{
Zentralbibliothek Zürich: räumliche Identität und Atmosphäre für Zürichs bibliophiles Paradies
}

\begin{abstract}
Der Wandel im Zeitalter der Digitalisierung bietet für die Zentralbibliothek räumliche Chancen. Der einst durch die analogen Kataloge beanspruchte Raum wird frei und durch die Zürcher Innenarchitekten von gasser, derungs reorganisiert und neu gestaltet. Die Innenarchitekten haben seit 2010 in drei Etappen verschiedene Bereiche des Altbaus, des Neubaus und des Predigerchors an die heutigen Bedürfnisse und Vorschriften angepasst. Dies jeweils in enger Zusammenarbeit mit der Bauherrschaft sowie der kantonalen Denkmalpflege. Entstanden sind in den knapp zehn Jahren des Umbaus zeitgemässe, einladende Arbeits-, Lern- und Ausstellungsorte, in denen Alt und Neu verschmelzen und die der geschichtsträchtigen Institution ein repräsentatives und freundliches Antlitz verleihen.
\end{abstract}

\section{Einführung}

Der argentinische Autor Jorge Luis Borges sagte einmal, dass er sich das Paradies immer als eine Art Bibliothek vorgestellt habe. Alle, die wie er eine Leidenschaft für Bücher teilen, werden ihm Recht geben. So auch die beiden Innenarchitekten Carmen Gasser Derungs und Remo Derungs, die selbst eine beachtliche Anzahl Bücher besitzen und die seit 2010 in drei Etappen Zürichs bibliophiles Paradies umgebaut, eingerichtet und an die Digitalisierung angepasst haben. Vor allem aber hat das Duo mit seinem Büro gasser, derungs Innenarchitekturen die von ihnen gestalteten öffentlichen Räume zu einem Ort des Wohlbefindens gewandelt. Die verschiedenen Ebenen und Ecken des Lesesaals wirken nun fast wie eine gehobene, gemütliche und zeitgemässe Hotellobby. Man tritt ein und fühlt sich willkommen.

Nebst den augenfälligen Renovationen und Sanierungen haben die Innenarchitekten auch einen grossen Teil an Arbeiten geleistet, die das Publikum kaum wahrnehmen wird: Sie betreffen die Sanierung von Erschliessungswegen im ganzen Gebäude, von Leitungen und technischen Installationen in enger Zusammenarbeit mit der Denkmalpflege.

Die Arbeitsweise von Carmen Gasser Derungs und Remo Derungs versteht und erlebt man sogleich, wenn man das Paar und sein Team in seinem Zürcher 
Büro besucht. Man tritt über die Schwelle des grossräumigen Arbeitsraums im Erdgeschoss eines Neubaus im Kreis 4 und befindet sich gleich mitten in dieser für sie typischen gastfreundlichen und gepflegten Atmosphäre. Wie in der Zentralbibliothek trifft man in ihrem Büro auf Arbeitsplätze, an die man sich gleich setzen möchte. Was die Innenarchitekten in ihrem Büro im Kleinen an Stimmung und Identität erschaffen haben, findet man der Situation und der Institution entsprechend auch am Predigerplatz wieder. Bezeichnend für das Vorgehen von gasser, derungs sind drei Punkte: das Erkennen des Potenzials eines Orts, mit dem Einsatz weniger Mittel überzeugende und ansprechende Lösungen zu finden sowie allen Räumlichkeiten etwas Einladendes zu verleihen.

\section{Etappe: die Reorganisation des Katalog- und Lesesaals}

Durch die Aufhebung des Zettelkataloges im Zeitalter der Digitalisierung entstand Raum in der seit ihrer Gründung immer wieder unter Platzknappheit leidenden Bibliothek. Den frei gewordenen Platz im Erdgeschoss des 1995 eröffneten Erweiterungsbaus haben die Innenarchitekten neu organisiert. Seit der Fertigstellung der ersten Etappe im Jahr 2010 stehen dort nun Bildschirmstationen, an denen Bücher und Informationen recherchiert und gegebenenfalls bestellt bzw. reserviert werden können. Zudem befinden sich dort verschiedene Arten von Arbeitsplätzen, eine Leselounge und seit diesem Sommer gleich beim Eingang in den Lesesaal die neue halbrunde Infotheke sowie eine zweite Lounge mit kleinen Tischen und Stehleuchten. Die diversen Bereiche werden durch verschiedenfarbiges Linoleum in dezenten Tönen gefasst und markiert. Schrittweise wurde auch ein Teil der Obergeschosse mit den neuen Arbeitsplätzen und dem neuen Boden ausgestattet.

Die neuen Einzelarbeitsplätze bestehen aus einem modularen System, sind alle mit Strom- und Internetanschlüssen ausgestattet und können beliebig lang aneinandergereiht werden. Die Arbeitsplätze bestehen aus einem mit schwarzem Linoleum belegten Tisch, der dreiseitig von Textilwänden in diversen Blautönen eingefasst wird, die dem Arbeitsplatz etwas Wohnliches verleihen, aber auch eine akustische Aufgabe erfüllen. Eine kleine Leuchte oben links beleuchtet die intime Arbeitsnische individuell. Schaut man vom Eingang in Richtung der Arbeitsplätze, wirken die streng gegliederten Reihen der Arbeitstische wie ein Muster aus blauen Vierecken.

Ergänzend zu den blauen Arbeitsplätzen haben die Innenarchitekten die auf vier Seiten von hohen Textilwänden umfassten grünen Cubicles entworfen. Diese 
Einzelkabinen sind neu in der Zentralbibliothek und werden rege genutzt. Aller Digitalisierung zum Trotz dienen diese Textilwände den Studierenden noch ganz analog als Pinnwände.

In der Mitte des Erdgeschosses hat gasser, derungs die neue Leselounge eingerichtet. Rund um die dunkelroten Sofas stehen Regale gefüllt mit Zeitungen und Zeitschriften zum Lesen. Eine imposante, kuppelartige Leuchte des Designers Ingo Maurer markiert den Treffpunkt, von wo aus man auf den Sofas sitzend bis unters Dach schauen kann. Im Herzen des Hauses darf sogar leise gesprochen werden.

\section{Etappe: die Wiederentdeckung des Hermann Escher-Saals}

Es ist heute kaum zu glauben, dass der Hermann Escher-Saal - einer der schönsten Räume im Altbau und gleich beim Eingang gelegen - über lange Zeit mit Schliessfächern verstellt war. Die Qualitäten des hohen Saales mit seinen schön gearbeiteten Stuckelementen und dem Fischgratparkett waren nicht mehr zu sehen. Das Potenzial des Ortes lag bis zum Umbau durch gasser, derungs brach. Es bedurfte einiger Überzeugungsarbeit seitens der Innenarchitekten, die Bauherrschaft dafür zu gewinnen, dass dieser repräsentative Saal als Veranstaltungsund Seminarraum genutzt und die Schliessfächer im Korridor des Untergeschosses untergebracht werden sollten.

In enger Zusammenarbeit mit der Denkmalpflege haben die Innenarchitekten den Hermann Escher-Saal saniert und dafür auch eine neue Leuchte entworfen, die Bezug zu einem historischen Exemplar im Haus aufnimmt. Entwickelt haben sie die Leuchte zusammen mit dem Zürcher Lichtplaner Michael J. Heusi. Entstanden ist nun ein Ort von hohem Aufenthalts- und Repräsentationswert, der für Vorträge für bis zu 100 Personen genutzt werden kann.

In der gleichen Etappe wurden im Korridor des Untergeschosses die neuen Schliessfächer geschaffen. Die gelbe Farbgebung der Schliessfächer und Wände verleihen dem durch Liftschächte und Treppen unterteilten Ort eine zusammenhängende Identität. Ebenfalls im Untergeschoss sind drei weitere Seminarräume entstanden.

Beim Eingang im Erdgeschoss haben die Planer den Raum für die Ausleihe und Rückgaben mit einfachen Mitteln zu einem repräsentativen Ort gewandelt. Sie haben hier einen Fischgratparkett verlegt, die Wände des hohen Raumes bis zur Hälfte in dunkelrote Farbe getaucht und die Regale und Informationstheke in dunklem Anthrazit gestaltet. Ein schönes Detail ist die frei im Raum hängende 
Signaletik aus einzelnen Buchstaben, welche die Reservationen und die Ausleihe markiert. Auch hier sind es klare und einfache Gestaltungselemente, die dem Raum seine Identität und seinen repräsentativen Status verleihen.

\section{Etappe: das modulare Ausstellungssystem der Schatzkammer}

Parallel zum Alt- und Neubau der Zentralbibliothek befindet sich - über diverse Bauten verbunden - die Predigerkirche mit ihrem mächtigen Chor. Dieser wurde bereits vor Jahrhunderten vom Hauptschiff abgetrennt, mit Zwischenböden ausgestattet und anderweitig genutzt. Seit einiger Zeit dienen die oberen Räume des Chors nun der Musikaliensammlung der Zentralbibliothek und das Erdgeschoss mit seiner Schatzkammer als Ausstellungsraum.

Die Erschliessungssituation des Chors hat das Zürcher Architekturbüro von Oliver Menzi und Philippe Bürgler grundlegend erneuert und mit einem einladenden Entrée aufgewertet. Nun gelangen die Besucher über eine repräsentative Aussentreppe aus dem Bibliothekshof direkt in das Foyer mit dem Empfangsbereich. Von hier tritt man über eine Tür in die allzeit verdunkelte Schatzkammer, die gasser, derungs neu gestaltet hat. Zusammen mit Menzi Bürgler Architekten haben sie entschieden, das Stützenraster des Raumes - die erste Betonskelettkonstruktion Zürichs - freizulegen und zu betonen. Auch die Materialien haben die beiden Büros gemeinsam ausgewählt. Zum Einsatz kommen beim Umbau des Predigerchors Eichenholz, Edelputz, Beton und Messing. Materialien, die mit der Zeit patinieren und sich der Materialität des historischen Baus angleichen. So findet man diese auch in der Schatzkammer. In die vorhandenen Wandnischen wurden mit Messingrahmen eingefasste Vitrinen integriert, während in der Raummitte modular aufgebaute Inseln und aus Zürcher Eiche gefertigte Ausstellungsinseln entstanden. Diese bestehen aus Tischvitrinen, Ganzglasvitrinen, Audiound Videostationen oder Sitzpolstern. Diese Inseln können je nach Ausstellungskonzept individuell aufgebaut und umplatziert werden. Ergänzend dazu haben die Innenarchitekten Stellwände für Texte und Bilder entworfen. Entlang den Wänden verkleidet eine aus Eichenstäben fabrizierte Brüstung die Radiatoren.

In der Schatzkammer wie auch im Lesesaal und im Herman Escher-Saal lassen die Innenarchitekten Alt und Neu zu einer Einheit verschmelzen. Die Geschichte des Ortes bleibt sicht- und erlebbar - ein Mehrwert auch im Zeitalter der Digitalisierung. 


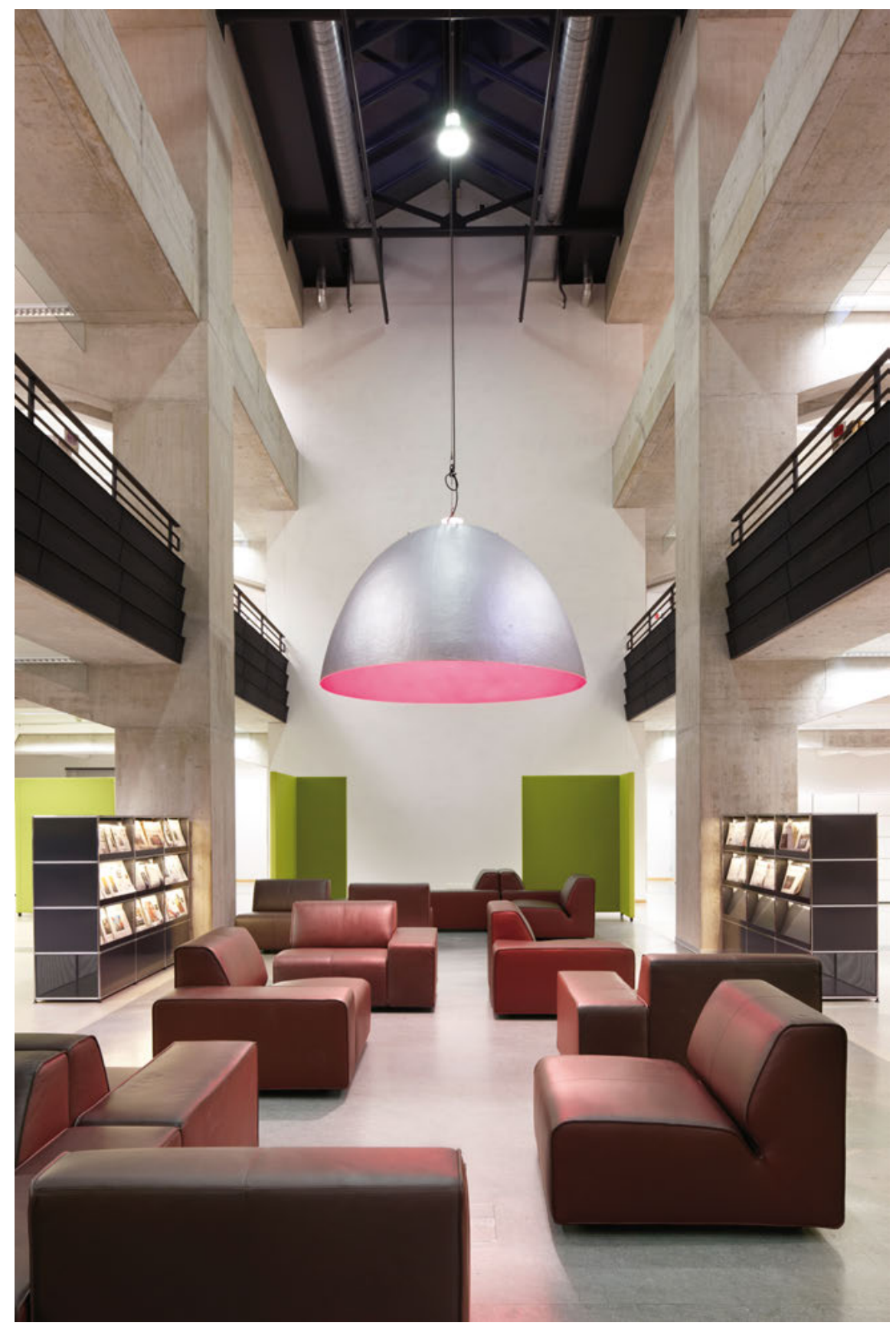

Abb. 1: Die Leselounge ist das Herz des Lesesaals. (@ Ralph Feiner) 

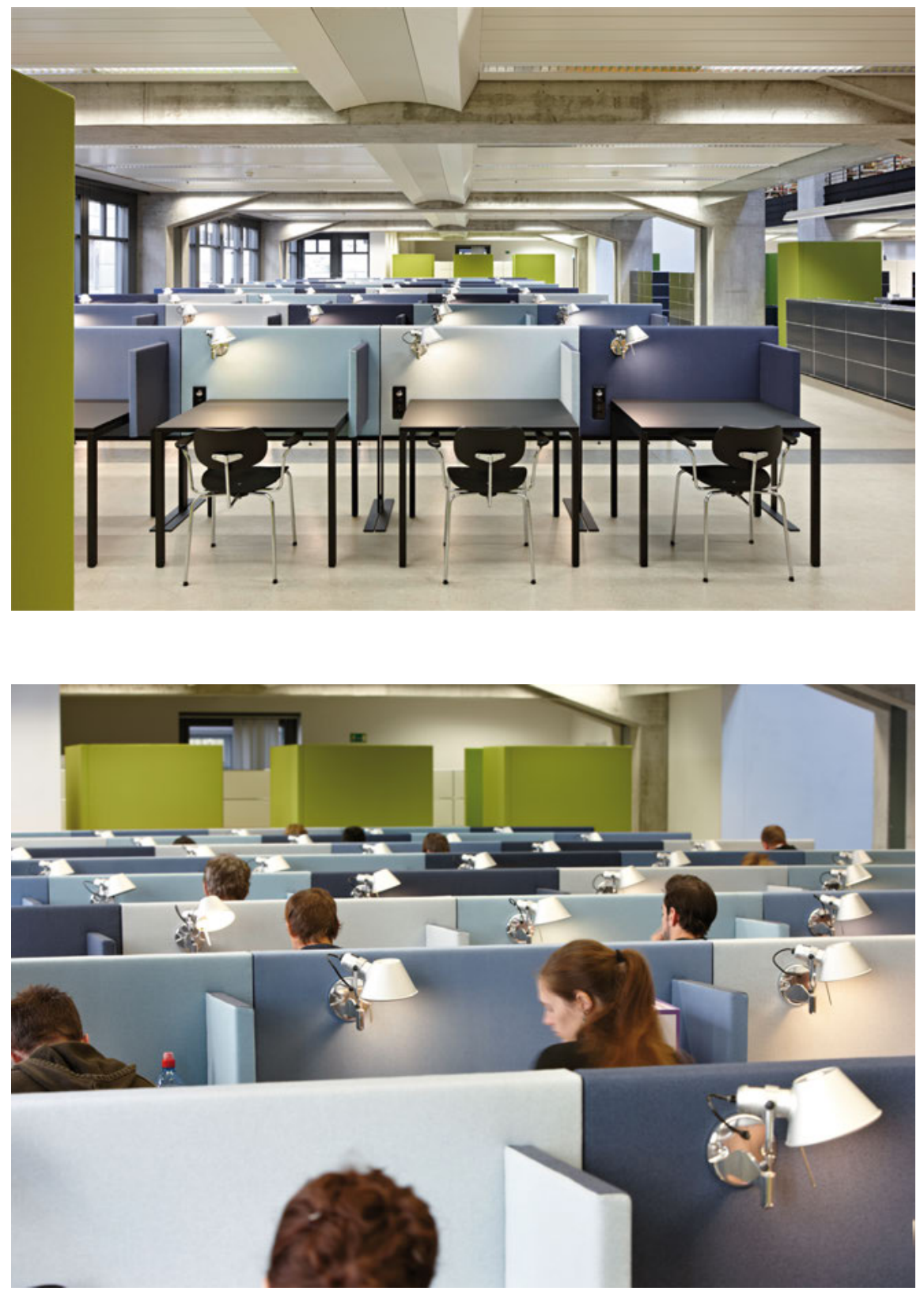

Abb. 2 und 3: Die neuen Arbeitsplätze bilden ein blaues Muster im Raum. (@ Ralph Feiner) 


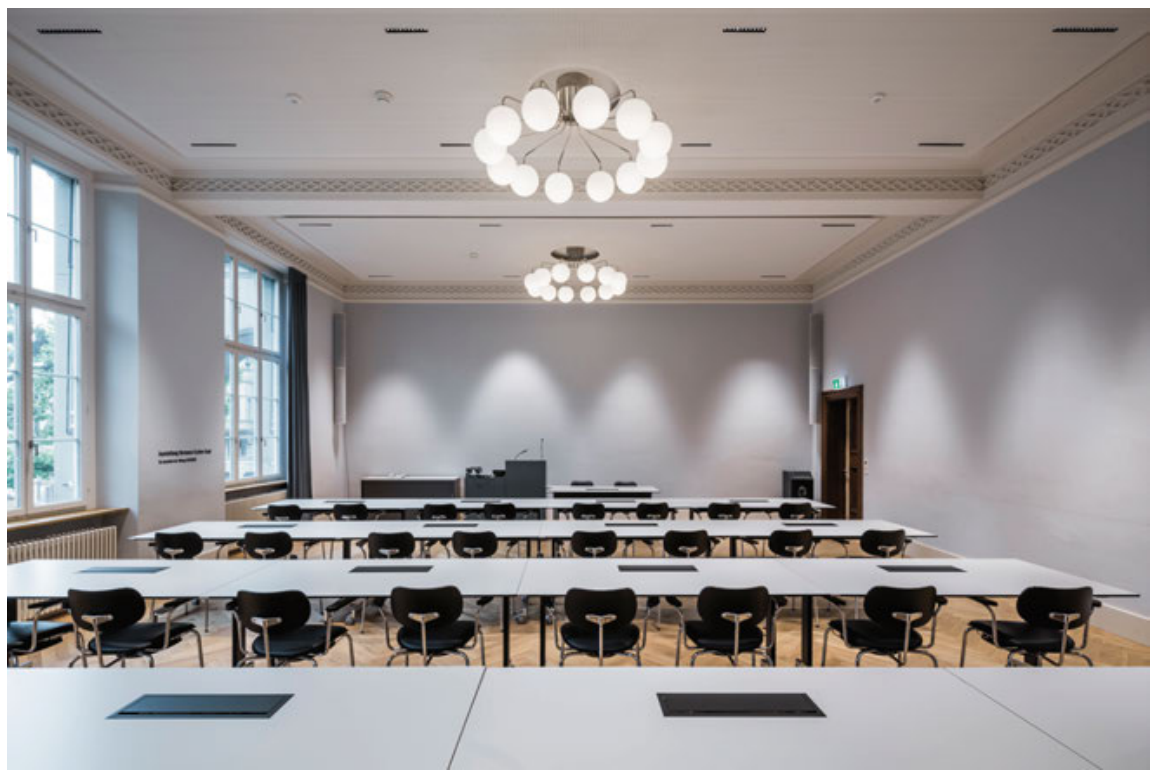

Abb. 4: Der historische Hermann Escher-Saal dient als Vortragsraum für bis zu 100 Personen. (C Benjamin Hofer)

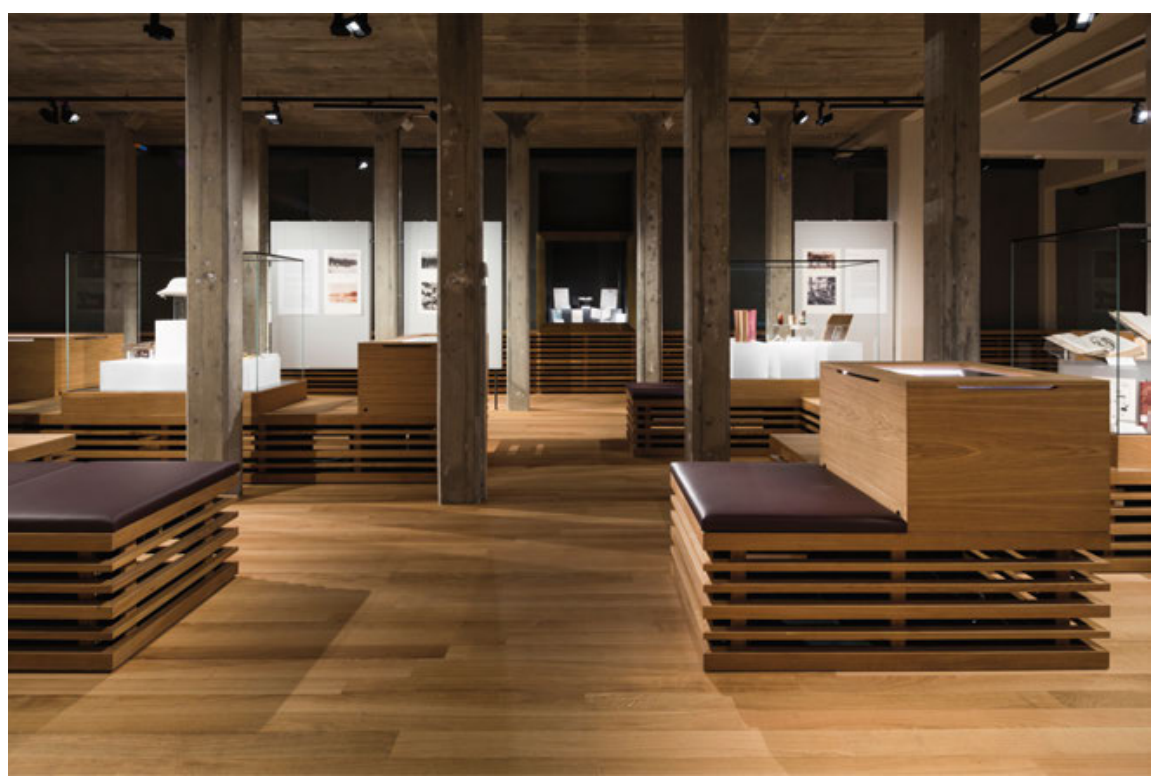

Abb. 5: Mit einem modularen Ausstellungssystem können wertvolle Exponate in der Schatzkammer inszeniert werden. (@ Benjamin Hofer) 